\title{
\begin{tabular}{l|l} 
Mibraries & DSpace@MIT
\end{tabular}
}

\author{
MIT Open Access Articles
}

\section{NUCLEOSYNTHESIS IN A PRIMORDIAL SUPERNOVA: CARBON AND OXYGEN ABUNDANCES IN SMSS J031300.36-670839.3}

The MIT Faculty has made this article openly available. Please share how this access benefits you. Your story matters.

Citation: Bessell, Michael S., Remo Collet, Stefan C. Keller, Anna Frebel, Alexander Heger, Andrew R. Casey, Thomas Masseron, et al. “NUCLEOSYNTHESIS IN A PRIMORDIAL SUPERNOVA: CARBON AND OXYGEN ABUNDANCES IN SMSS J031300.36-670839.3." The Astrophysical Journal 806, no. 1 (June 8, 2015): L16. (C) 2015 The American Astronomical Society

As Published: http://dx.doi.org/10.1088/2041-8205/806/1//16

Publisher: IOP Publishing

Persistent URL: http://hdl.handle.net/1721.1/98372

Version: Final published version: final published article, as it appeared in a journal, conference proceedings, or other formally published context

Terms of Use: Article is made available in accordance with the publisher's policy and may be subject to US copyright law. Please refer to the publisher's site for terms of use. 


\title{
NUCLEOSYNTHESIS IN A PRIMORDIAL SUPERNOVA: CARBON AND OXYGEN ABUNDANCES IN SMSS J031300.36-670839.3*
}

\author{
Michael S. Bessell ${ }^{1}$, Remo Collet ${ }^{1}$, Stefan C. Keller ${ }^{1,9}$, Anna Frebel ${ }^{2}$, Alexander Heger ${ }^{3,10,11}$, Andrew R. Casey ${ }^{4}$, \\ Thomas Masseron ${ }^{4}$, Martin Asplund ${ }^{1}$, Heather R. Jacobson ${ }^{5}$, Karin Lind ${ }^{6}$, Anna F. Marino ${ }^{1}$, John E. Norris ${ }^{1}$, \\ David Yong ${ }^{1}$, Gary Da Costa ${ }^{1}$, Conrad Chan $^{3}$, Zazralt Magic ${ }^{7}$, Brian Schmidt ${ }^{1}$, and Patrick Tisserand ${ }^{8,12}$ \\ ${ }^{1}$ Research School of Astronomy and Astrophysics, CPMS, The Australian National University, Canberra, ACT 2611, Australia; michael.bessell@anu.edu.au, \\ remo.collet@anu.edu.au, stefan.keller@defence.gov.au, martin.asplund@anu.edu.au, anna.marino@anu.edu.au, \\ john.norris@anu.edu.au, david.yong@anu.edu.au, gary.dacosta@anu.edu.au, brian.schmidt@ anu.edu.au \\ ${ }^{2}$ Department of Physics and Kavli Institute for Astrophysics and Space Science, Massachusetts Institute of Technology, \\ Cambridge, MA 02139, USA; afrebel@mit.edu \\ ${ }^{3}$ School of Physics and Astronomy, Monash University, VIC 3800, Australia; alexander.heger@monash.edu, conrad.chan@monash.edu \\ ${ }^{4}$ Institute of Astronomy, University of Cambridge, Cambridge CB3 0HA, UK; arc@ast.cam.ac.uk, tpm40@ast.cam.ac.uk \\ ${ }^{5}$ Kavli Institute for Astrophysics and Space Science, Massachusetts Institute of Technology, Cambridge, MA 02139, USA; hrj@mit.edu \\ ${ }^{6}$ Department of Physics and Astronomy, Uppsala University, Box 516, SE-751 20, Uppsala, Sweden; karin.lind@physics.uu.se \\ ${ }^{7}$ Max-Planck-Institut für Astrophysik, D-85741 Garching, Germany; magic@ MPA-Garching.MPG.DE \\ ${ }^{8}$ Sorbonne Universités, UPMC Univ Paris 06, UMR 7095, Institut d'Astrophysique de Paris, F-75014 Paris, France; tisserand@iap.fr \\ ${ }_{9}^{9}$ Department of Defense, Australian Government, Canberra, ACT 2610, Australia \\ ${ }^{10}$ School of Physics and Astronomy, University of Minnesota, Minneapolis, MN 55455, USA \\ ${ }^{11}$ CNA, Department of Physics and Astronomy, Shanghai Jiao-Tong University, Shanghai 200240, P. R. China \\ ${ }^{12}$ UPMC-CNRS, UMR7095, Institut d'Astrophysique de Paris, F-75014 Paris, France \\ Received 2015 March 13; accepted 2015 May 12; published 2015 June 8
}

\begin{abstract}
SMSS J031300.36-670839.3 (hereafter SM0313-6708) is a sub-giant halo star, with no detectable Fe lines and large overabundances of $\mathrm{C}$ and $\mathrm{Mg}$ relative to $\mathrm{Ca}$. We obtained Very Large Telescope-Ultraviolet and Visual Echelle Spectrograph (UVES) spectra extending to $3060 \AA$ showing strong OH A-X band lines enabling an oxygen abundance to be derived. The $\mathrm{OH}$ A-X band lines in SM0313-6708 are much stronger than the $\mathrm{CH}$ C-X band lines. Spectrum synthesis fits indicate an $[\mathrm{O} / \mathrm{C}]$ ratio of $0.02 \pm 0.175$. Our high signal-to-noise ratio UVES data also enabled us to lower the Fe abundance limit to $[\mathrm{Fe} / \mathrm{H}]_{\langle 3 \mathrm{D}\rangle, \mathrm{NLTE}}<-7.52(3 \sigma)$. These data support our previous suggestion that the star formed from the iron-poor ejecta of a single massive star Population III supernova.
\end{abstract}

Key words: stars: Population III - supernovae: general - supernovae: individual (SMSS J031300.36)

\section{INTRODUCTION}

Keller et al. (2014) described the discovery of SMSS J031300.36-670839.3 (hereafter SM0313-6708) and the analysis of the high resolution spectrum taken with the Magellan Inamori Kyocera Echelle (MIKE; Bernstein et al. 2003) spectrograph on the $6.5 \mathrm{~m}$ Magellan Telescope at Las Campanas Observatory in Chile. The spectrum was remarkable for the complete absence of lines of iron or any other metal, apart from $\mathrm{Mg}$ I and $\mathrm{Ca}$ II. The fact that no iron line is evident in the signal-to-noise ratio $(\mathrm{S} / \mathrm{N}) \sim 100$ (at $\lambda=4000 \AA$ ) spectrum indicated that the iron abundance in SM0313-6708 was at most $[\mathrm{Fe} / \mathrm{H}]=-7.2,{ }^{13}$ making this star at least $\sim 40$ times more iron-poor than HE 1327-2326 $([\mathrm{Fe} / \mathrm{H}]=-5.6$; Frebel et al. 2005).

The only elements whose abundances could be measured were lithium, carbon, magnesium, and calcium with $A(\mathrm{Li})$ $=12+\log (\mathrm{Li} / \mathrm{H})=0.71 \pm 0.10, \quad[\mathrm{C} / \mathrm{H}]=-2.44 \pm 0.10$, $[\mathrm{Mg} / \mathrm{H}]=-4.30 \pm 0.10$, and $[\mathrm{Ca} / \mathrm{H}]=-7.2 \pm 0.10$, respectively. The level of lithium is presumably related to the decline from the primordial Spite plateau level of $A(\mathrm{Li})=2.2$ during early post-main-sequence stellar evolution (Lind et al. 2009) and this evidence, together with a $T_{\text {eff }}=5125 \mathrm{~K}$ and $\log g=2.3$ derived from spectrophotometry, is consistent with

\footnotetext{
* Based on observations obtained with European Southern Observatory (ESO) telescopes (proposal 092.D-0742).

${ }^{13}$ Logarithm of abundance ratio relative to present day solar surface abundances, $[\mathrm{A} / \mathrm{B}]=\log \left(n_{\mathrm{A}} / n_{\mathrm{B}}\right)-\log \left(n_{\mathrm{A}} / n_{\mathrm{B}}\right)_{\odot}$.
}

a star ascending the giant branch for the first time. The $\mathrm{C}, \mathrm{Mg}$, and $\mathrm{Ca}$ abundances can be attributed to a low energy Pop III $\mathrm{SNe}$, and measurement of the oxygen abundance, in particular, offers an important test of this scenario. To measure the oxygen abundance in ultra-metal-poor $\mathrm{K}$ stars, however, it is necessary to work in the ultraviolet and observe the $\mathrm{OH}$ lines that persist well after the [O I] $6300.3 \AA$ line has become undetectable.

\section{OBSERVATIONS}

In order to measure the $\mathrm{OH}$ (and $\mathrm{CH}$ ) lines between 3100 and $3200 \AA$ and obtain better detection limits on N, Na, V, Co, $\mathrm{Ni}, \mathrm{Fe}$, and $\mathrm{Cu}$, we used Ultraviolet and Visual Echelle Spectrograph (UVES; Dekker et al. 2000) on the VLT (ESO Very Large Telescope) at Paranal in Chile. Between 2013 and 2014 September, 31 exposures totalling $29.26 \mathrm{hr}$ of on-source integration were obtained. The UVES UV spectrum extended from 3060 to $3860 \AA$ and the two red spectra from 4786 to $6803 \AA$.

The data were reduced using the default settings of the UVES pipeline as implemented in the "reflex" reduction environment. There was a high order sinusoidal-like modulation of the continuum remaining in much of the UV spectrum. The modulation with an amplitude of about $20 \%$ and frequency about $50 \AA$ was removed by hand, leaving a residual variation of amplitude of $2 \%-4 \%$ and spacing of about $1.5 \AA$ that makes the measurement of shallow features somewhat uncertain. It was very clear, however, that there were many strong $\mathrm{OH}$ lines present between the observational cutoff around 3060 and 


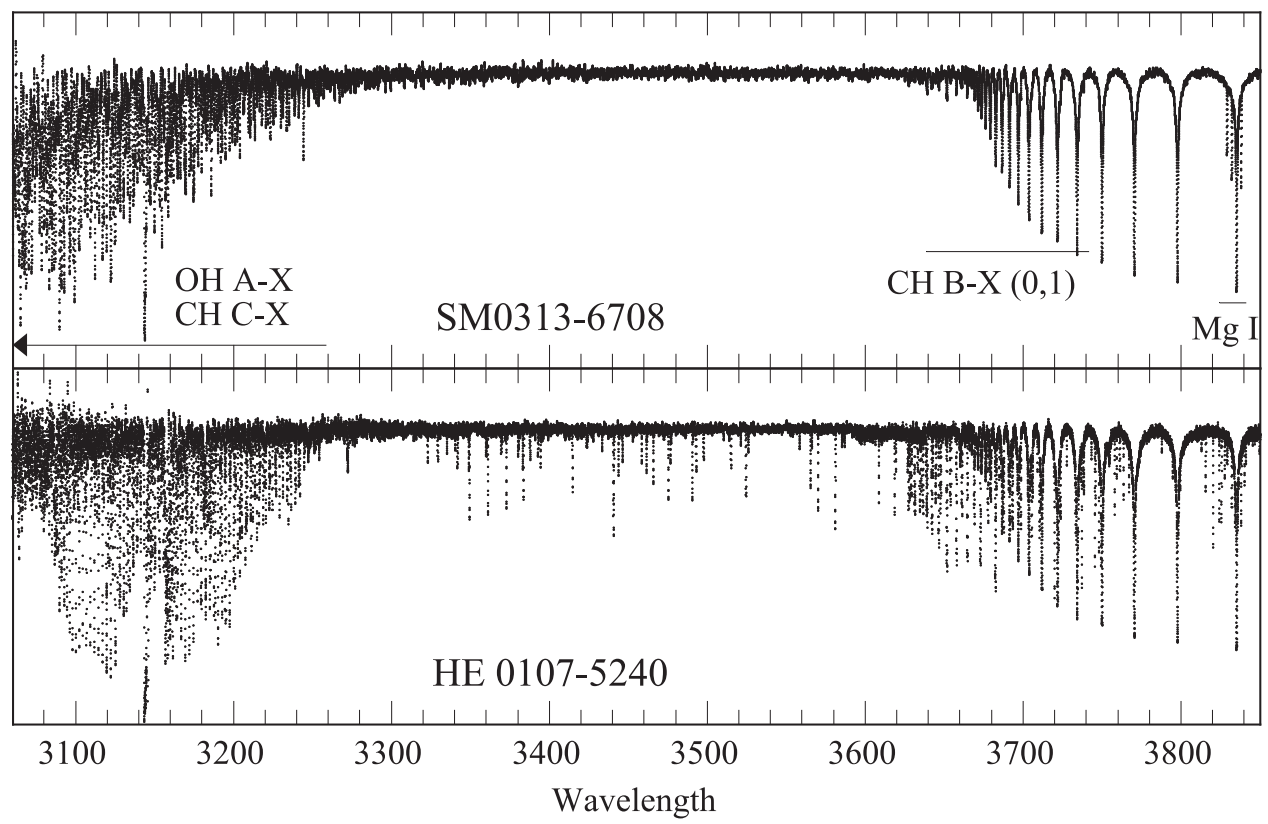

Figure 1. Normalized UVES UV spectra of SM0313-6708 and HE 0107-5240. Note the different distribution of the OH and CH lines in the two stars and the complete absence of metal lines in SM0313-6708 except for the similar strength Mg I lines.

$3200 \AA$, and that the $\mathrm{OH}$ lines were much stronger than the $\mathrm{CH}$ lines, except at the band head of the CH C-X band near $3144 \AA$. In Figure 1 we compare the UV spectra of SM0313-6708 $([\mathrm{Fe} / \mathrm{H}] \leqslant-7.2)$ and $\mathrm{HE} 0107-5240([\mathrm{Fe} / \mathrm{H}]=-5.4)$, a C-rich ultra-EMP star with almost identical effective temperature and gravity, and possibly at the same evolutionary stage. The total absence of metal lines in SM0313-6708 (except for the $\mathrm{Mg}$ I lines around $3830 \AA$ ) is very marked as is the different distribution of the forest of molecular lines below $3250 \AA$. The $\mathrm{CH}$ lines dominate the spectrum of HE 0107-5240 whereas $\mathrm{OH}$ lines dominate in SM0313-6708. The complete absence of absorption lines in SM0313-6708 between 3250 and $3800 \AA$ enables more stringent limits to be placed on those species with strong lines in that region.

\section{ANALYSIS}

We have carried out a spectrum synthesis of the $\mathrm{CH}, \mathrm{OH}$, and NH molecular lines in SM0313-6708 using Turbospectrum (Alvarez \& Plez 1998; Plez 2012). We adopted an interpolated "standard" metallicity MARCS 1D LTE model atmosphere structure (Gustafsson et al. 2008), for $T_{\text {eff }}=5125$ $\mathrm{K}, \log g=2.30,[\mathrm{M} / \mathrm{H}]=-5.0$, but with actual abundances from this paper for the atomic and molecular equilibria calculations. We used the new ${ }^{12} \mathrm{CH}$ and ${ }^{13} \mathrm{CH}$ line list from Masseron et al. (2014), while for $\mathrm{OH}$ and $\mathrm{NH}$ we used extensive line lists recently recomputed by T. Masseron (2015, in preparation). The Masseron $\mathrm{OH} g f$ values are very similar to those of Gillis et al. (2001), but the $\mathrm{NH}$ of values are significantly smaller by $0.3-0.7$ dex than the commonly used values of Kurucz (2011). The solar composition was adopted from Asplund et al. (2009).

We refitted the $\mathrm{CH} A-\mathrm{X}$ and $\mathrm{B}-\mathrm{X}$ lines in the blue MIKE spectrum and determined a carbon abundance of $[\mathrm{C} / \mathrm{H}]=-2.41 \pm 0.05$, very similar to the previously determined A-X value. The $\mathrm{CH} C-\mathrm{X}$ band synthesis is in good agreement with the A-X and $\mathrm{B}-\mathrm{X}$ bands. The synthesized spectrum well reproduces the observed $\mathrm{CH}$ lines in strength and position for all $J$ values and for all branches observed.

The $\mathrm{OH}$ lines extend from 3060 to redward of $3200 \AA$. Because of the lower $\mathrm{S} / \mathrm{N}$ and the uncertain continuum placement at the shortest wavelengths where the lines are strongest, we derived the oxygen abundance in SM0313-6708 by fitting the weaker $\mathrm{OH}$ lines between 3178 and $3200 \AA$. Figure 2 clearly shows that the oxygen abundance is well constrained at $[\mathrm{O} / \mathrm{H}]=-2.00 \pm 0.10$. In HE 0107-5240, the similar temperature and gravity star with abundances $[\mathrm{M} / \mathrm{H}] \approx-5.4,[\mathrm{C} / \mathrm{H}]=-1.58$ (Christlieb et al. 2004) and $[\mathrm{O} / \mathrm{H}]=-3.0$ (Bessell et al. 2004), these longer wavelength regions could not be used for $\mathrm{OH}$ fitting as they are dominated by $\mathrm{CH}$ lines as seen in Figure 3. We note that the treatment of scattering (as done in Turbospectrum), is very important for the analysis of the UV lines in SM0313-6708 and metal-poor giants in general.

Figure 3 shows the comparison between the observed and synthetic spectra at the shorter wavelengths in SM0313-6708 and HE 0107-5240. The panels on the left show the region that was used to measure the oxygen abundance in HE 0107-5240, and the panels on the right shows the region around the $\mathrm{CH}$ $\mathrm{C}-\mathrm{X}$ band head. Note the significantly different relative strengths of $\mathrm{OH}$ and $\mathrm{CH}$ lines in the two stars.

There is no indication of any $\mathrm{NH}$ features centered on $3360 \AA$, but the higher $\mathrm{S} / \mathrm{N}$ of the UVES spectrum enables us to reduce the upper limit to $[\mathrm{N} / \mathrm{H}]<-4.2$, at least two orders of magnitude lower than $\mathrm{C}$ and $\mathrm{O}$.

\section{1. $3 D-1 D$ Corrections to $C$ and $O$ Abundances}

The carbon, nitrogen, and oxygen abundances reported above were derived from 1D LTE analysis of the $\mathrm{CH}$ and $\mathrm{OH}$ lines. One of us (RC) generated a 3D time-dependent hydrodynamic model atmosphere of a red giant star with stellar parameters very similar to the ones of SM0313-6708 using a custom version of the STAGGer code (Nordlund \& Galsgaard 1995; Nordlund et al. 2009) as well as a 1D model 

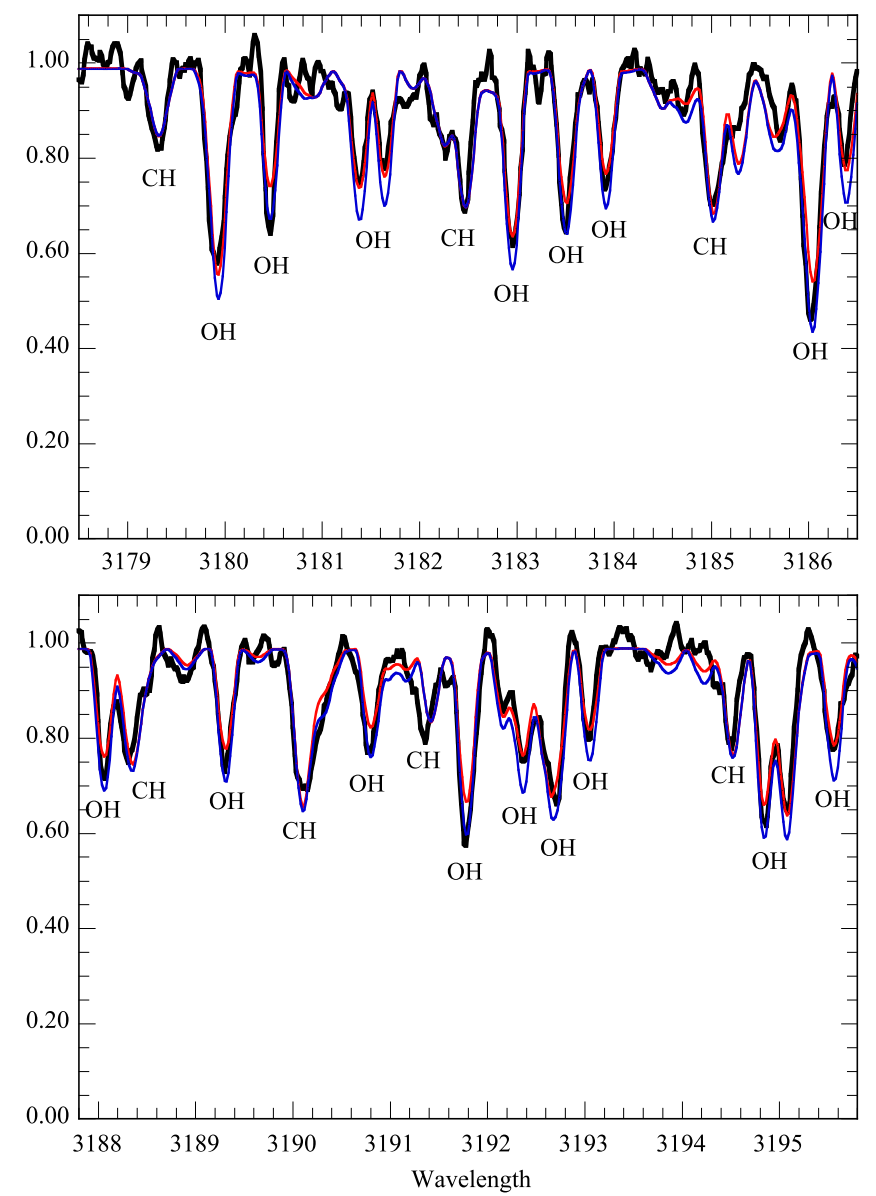

Figure 2. Region between 3178 and $3196 \AA$ in SM0313-6708. Observations (thick black line); synthetic spectra for $[\mathrm{C} / \mathrm{H}]=-2.41$ and oxygen abundance $[\mathrm{O} / \mathrm{H}]=-2.10$ (red line) and higher abundance $[\mathrm{O} / \mathrm{H}]=-1.90$ (blue line). Although the $\mathrm{OH}$ lines here are weaker than the 3060-3200 A region, the S/N is higher and the continuum level is better defined.

atmosphere corresponding to the same parameters and relying on the same microphysics and opacity data. Synthetic LTE CH and $\mathrm{OH}$ spectra for a range of $\mathrm{C}$ and $\mathrm{O}$ abundances were produced using the SCATE code (Hayek et al. 2011) with 3D and 1D models, accounting for the effects of radiative scattering; the best fits to the observed spectrum were determined in both cases and the 3D-1D abundance corrections were then derived. These $3 \mathrm{D}-1 \mathrm{D}$ corrections are: $-0.14 \mathrm{dex}$ for the carbon abundance as derived from the $\mathrm{CH}$ band and -0.53 dex for oxygen from $\mathrm{OH}$ (the negative sign meaning the $3 \mathrm{D}$-derived abundances are lower). The 3D-corrected $[\mathrm{O} / \mathrm{C}]$ ratio in SM0313-6708 is therefore +0.02 with an uncertainty of 0.175 dex. A 3D-1D correction of -0.50 dex to the $1 \mathrm{D}$ nitrogen abundance was also derived with similar 3D and 1D LTE calculations but based purely on the comparison of synthetic $3 \mathrm{D}$ and $1 \mathrm{D}$ spectra.

The poorer fit of the deep $\mathrm{OH}$ and $\mathrm{CH}$ lines in Figure 3 is likely due to limitations with the adopted 1D model atmosphere. Our computations of $\mathrm{CH}, \mathrm{NH}$, and $\mathrm{OH}$ lines have been carried out in LTE, but in low-density atmospheres it is possible that non-LTE processes may be important for cooling the outer stellar layers (Lambert et al. 2013) and for affecting the strength of molecular bands. As no study of non-LTE CH, $\mathrm{NH}$, and $\mathrm{OH}$ line formation is available in the literature, however, we are unable to quantify non-LTE effects on CNO abundances derived from these molecular bands.

$$
\text { 3.2. }{ }^{12} \mathrm{Cl}^{13} \mathrm{C} \text { Ratio }
$$

In order to put limits on the ${ }^{12} \mathrm{C} /{ }^{13} \mathrm{C}$ ratio we used two regions of the spectrum where the ${ }^{12} \mathrm{CH}$ and ${ }^{13} \mathrm{CH}$ lines are well separated and free from blending. There were five strong lines in the B-X region and nine in the A-X region. Sections of spectrum centered on each of the lines were extracted and summed in both the observed spectrum and the synthetic spectra. The summed observed and synthetic ${ }^{12} \mathrm{CH}$ lines were in good agreement, but no ${ }^{13} \mathrm{CH}$ feature was evident in the summed spectrum. This allows us to place a three sigma lower limit of about 40 for the ${ }^{12} \mathrm{C} /{ }^{13} \mathrm{C}$ ratio. This low value of ${ }^{13} \mathrm{C}$ is consistent with massive Population III star yields with no or little primary nitrogen production (Heger \& Woosley 2010).

\subsection{Revised Upper Limits on the Abundances of $\mathrm{Fe}, \mathrm{Na}, \mathrm{Si}, \mathrm{Sc}, \mathrm{V}, \mathrm{Co}, \mathrm{Ni}, \mathrm{Cu}$}

Two of the Fe I transitions with the largest $\log g f$ values in the observed spectral range are located within the highest $\mathrm{S} / \mathrm{N}$ portion of the UV spectrum, at 3719.94 and $3737.13 \AA$. Sections around the position of these lines were extracted, summed and compared with the summed synthetic spectra computed for a range of $\mathrm{Fe}$ abundances. Again, no feature was evident in the observed spectrum, and we estimate a revised abundance limit for $[\mathrm{Fe} / \mathrm{H}]$ of about -7.5.

The non-detection of lines of $\mathrm{Sc}, \mathrm{Ti}, \mathrm{V}, \mathrm{Co}, \mathrm{Ni}$, and $\mathrm{Cu}$ in the UVES spectrum in the range from 3100 to $3700 \AA$ provided revised lower upper limits for many elements given in Table 1 (A. Frebel et al. 2015, in preparation). The non-detection of the Si I line at $3905.52 \AA$ in the blue MIKE spectrum indicates an upper limit of -5.6 dex.

The UVES red spectrum was in good agreement with the MIKE spectrum for the $\mathrm{Mg}$ I lines and the $\mathrm{Li}$ I line, but the better $\mathrm{S} / \mathrm{N}$ and weaker telluric lines in the UVES red spectrum indicates that the $\mathrm{Na}$ limit could be lowered to about -5.7 dex. Table 1 lists the current best estimates for the chemical abundances of SM0313-6708.

\section{DISCUSSION}

Heger \& Woosley (2010) have followed the evolution and explosion of metal-free stars with masses $10 M_{\odot}-100 M_{\odot}$ and determined their nucleosynthetic yields, light curves, and remnant masses. Such stars would have been the first to form after the Big Bang and could have left a distinctive imprint on the composition of the early universe. The evolution of these stars may be affected in several ways by their zero-metallicity primordial composition. Because these stars suffered little mass-loss during their pre-explosive lifetimes, their masses at their deaths were larger than more metal-rich stars. The models produces some carbon, but for masses less than about $40 M_{\odot}$ primary production of nitrogen is suppressed. These stars ended their lives as compact blue supergiants making their envelope more tightly bound, with two interesting consequences: (i) the more compact envelope increases the amount of mass that falls back after the explosion, meaning that they were more likely to collapse to black holes at lower masses compared to modern stars, trapping most of the heavy elements; (ii) mixing by the Rayleigh-Taylor instabilities is suppressed in these compact stars, so it is mainly unmixed 

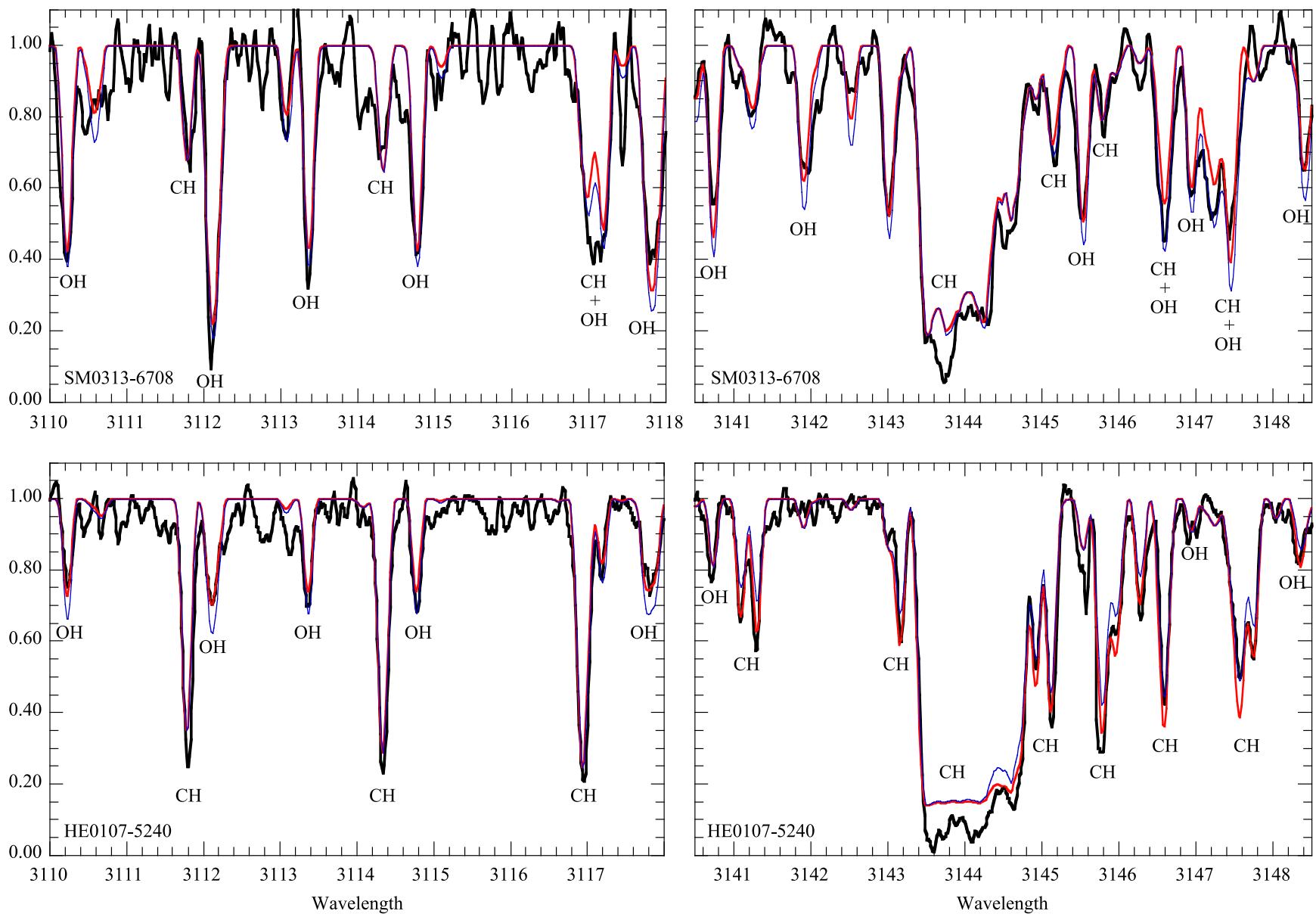

Figure 3. Spectral region showing mainly $\mathrm{OH}$ lines (left) and a region around the $\mathrm{CH} \mathrm{C}-\mathrm{X}$ band head (right). Top: SM0313-6708. Observations (thick black line); synthetic spectra for abundance $[\mathrm{O} / \mathrm{H}]=-2.10$ (red line) and higher abundance $[\mathrm{O} / \mathrm{H}]=-1.90$ (blue line). $[\mathrm{C} / \mathrm{H}]=-2.41$ in both syntheses. The $3110-3118 \AA$ region has low S/N and uncertain continuum placement. Bottom: HE 0107-5240. Observations (thick black line). Left: synthetic spectra for best-fitting abundances $[\mathrm{C} / \mathrm{H}]=-1.20,[\mathrm{O} / \mathrm{H}]=-2.55$ (red line) and higher abundance $[\mathrm{O} / \mathrm{H}]=-2.40$ (blue line). Right: $[\mathrm{O} / \mathrm{H}]=-2.55,[\mathrm{C} / \mathrm{H}]=-1.20($ red line) and lower abundance $[\mathrm{C} / \mathrm{H}]=-1.40$ (blue line).

material that escapes in the explosion. Heger \& Woosley (2010) fitted their predicted nucleosynthetic yields to several examples of metal-poor stars and found that the best fits were obtained from relatively low energy explosions of $0.6-1.8 \mathrm{~B}\left(1 \mathrm{~B}=10^{51} \mathrm{erg}\right)$ as compared to hypernova models (Nakamura et al. 2001). Overall, the large sample of metalpoor stars in Cayrel (2004) was well fit with a normal Salpeter IMF including all stars from $10 M_{\odot}$ to $100 M_{\odot}$ (but with a preference for stars lighter than $15 M_{\odot}$ ), a small amount of fallback and with reduced mixing of $1 \%$ of the helium core mass (for reference, the default mixing value adopted to solar metallicity stars is $10 \%$ of the helium core mass; Heger \& Woosley 2010). For the two ultra-iron-poor, but high $\mathrm{O} / \mathrm{Fe}$, stars HE 0107-5240 (Bessell et al. 2004; Christlieb et al. 2004) and HE 1327-2326 (Aoki et al. 2006; Frebel et al. 2006), the best fits were for similar low energy explosions, a lower upper mass range $\left(10 M_{\odot}\right.$ to less than $\left.30 M_{\odot}\right)$, small fallback and an even lower mixing of 0.025 .

The abundances of SM0313-6708 are much more extreme than the Cayrel (2004) EMP stars or the two C-rich ultra-EMP stars. The biggest difference are the large carbon, oxygen and magnesium overabundances relative to calcium, and the nondetection of all other metals. Of particular significance is the oxygen to carbon ratio in SM0313-6708 of $[\mathrm{O} / \mathrm{C}]_{3 \mathrm{D}}=+0.02$ compared to $[\mathrm{O} / \mathrm{C}]_{3 \mathrm{D}} \approx-1.0$ (Collet et al. 2006) in $\mathrm{HE}$ $0107-5240$.

Fitting the current SM0313-6708 abundances (Table 1) against models from Heger \& Woosley (2010) using their STARFIT algorithm ${ }^{14}$ finds good fits for progenitor masses in the range $40 M_{\odot}-60 M_{\odot}$ and energies of $1.5 \mathrm{~B}-1.8 \mathrm{~B}$ with low amounts of mixing, $\lesssim 2 \%$. This low amount of mixing is consistent with Heger \& Woosley (2010) and hydrodynamic models of Pop III SNe by Joggerst et al. (2009). The best matching mass is sensitive to the upper limits of the $\mathrm{Na}$ and $\mathrm{Al}$ abundances. Generally, lower values on the upper limits to the abundances would push the fit toward higher initial masses, stars that produce a stronger odd-even effect. The low limits on $\mathrm{N}$ in SM0313-6708 constrain the mass on the upper end.

The range of $[\mathrm{O} / \mathrm{C}]$ abundances produced in a range of core collapse supernovae is shown in Figure 4 together with a $40 M_{\odot}$ fit from StaRfit. Significantly, the observed $[\mathrm{O} / \mathrm{C}]$ ratio is lower than what Pop III pair-instability SNe of Heger \& Woosley (2002) can produce or what we can produce for high explosion energies $(E \gtrsim 3 \mathrm{~B})$ in more massive stars $\left(M \gtrsim 20 M_{\odot}\right)$. The structure and pre-SN evolution of stars with mass above $15 M_{\odot}$ is very sensitive to initial mass (e.g., Sukhbold \& Woosley 2014) and these stars may experience

\footnotetext{
${ }^{14}$ See also http://starfit.org (C. Chan et al. 2015, in preparation).
} 
Table 1

Chemical Abundances of SM0313-6708

\begin{tabular}{|c|c|c|c|c|c|}
\hline Element & {$[\mathrm{X} / \mathrm{H}]_{1 \mathrm{D}, \mathrm{LTE}}$} & {$[\mathrm{X} / \mathrm{H}]_{1 \mathrm{D}, \mathrm{NLTE}}$} & {$[\mathrm{X} / \mathrm{H}]_{\langle 3 \mathrm{D}\rangle, \mathrm{LTE}}$} & {$[\mathrm{X} / \mathrm{H}]_{\langle 3 \mathrm{D}\rangle, \mathrm{NLTE}}$} & {$[\mathrm{X} / \mathrm{H}]_{3 \mathrm{D}, \mathrm{LTE}}$} \\
\hline $\mathrm{C}(\mathrm{CH})^{\mathrm{b}}$ & $-2.41 \pm 0.05$ & $\ldots$ & $\ldots$ & $\ldots$ & $-2.55 \pm 0.09$ \\
\hline $\mathrm{O}(\mathrm{OH})^{\mathrm{b}}$ & $-2.00 \pm 0.10$ & $\ldots$ & $\ldots$ & $\ldots$ & $-2.53 \pm 0.15$ \\
\hline $\mathrm{Na}^{\mathrm{b}}$ & $<-5.7$ & $<-5.65$ & $<-5.79$ & $<-5.66$ & $\ldots$ \\
\hline $\mathrm{Mg}_{\mathrm{I}}^{\mathrm{b}}$ & $-4.45 \pm 0.07$ & $-4.03 \pm 0.03$ & $-4.53 \pm 0.09$ & $-4.08 \pm 0.03$ & $\ldots$ \\
\hline $\mathrm{Ca}$ II & $-7.46 \pm 0.10$ & $-7.27 \pm 0.10$ & $-7.60 \pm 0.10$ & $-7.26 \pm 0.10$ & $\ldots$ \\
\hline $\mathrm{Sc}$ II $^{\mathrm{b}}$ & $<-5.5$ & $\ldots$ & $\ldots$ & $\ldots$ & $\ldots$ \\
\hline $\mathrm{Ti}_{\mathrm{II}}^{\mathrm{b}}$ & $<-7.1$ & $\ldots$ & $\ldots$ & $\ldots$ & $\ldots$ \\
\hline $\mathrm{V}_{\text {II }}^{\mathrm{b}}$ & $<-4.3$ & $\ldots$ & $\ldots$ & $\ldots$ & $\ldots$ \\
\hline $\mathrm{Cr}_{\mathrm{I}}$ & $<-6.3$ & $\ldots$ & $\ldots$ & $\ldots$ & $\ldots$ \\
\hline Mn I & $<-5.8$ & $\ldots$ & $\ldots$ & $\ldots$ & $\ldots$ \\
\hline $\mathrm{Zn}$ I & $<-3.4$ & $\ldots$ & $\ldots$ & $\ldots$ & $\ldots$ \\
\hline Sr II & $<-6.9$ & $\ldots$ & $\ldots$ & $\ldots$ & $\ldots$ \\
\hline Ba II & $<-6.1$ & $\ldots$ & $\ldots$ & $\ldots$ & $\ldots$ \\
\hline Eu II & $<-2.9$ & $\ldots$ & $\ldots$ & $\ldots$ & $\ldots$ \\
\hline
\end{tabular}

Notes. Estimated $(1 \sigma)$ observational uncertainties in the quoted upper limit abundances are 0.3 dex. For Fe the uncertainty is $3 \sigma$. Bold print indicates our recommended values used in the fitting of explosive nucleosynthesis.

${ }^{a}$ The abundance of $\mathrm{Li}$ is expressed as $A(\mathrm{Li})=\log (\mathrm{N}(\mathrm{Li}) / \mathrm{N}(\mathrm{H}))+12$.

b Abundances derived in this paper.

chaotic nuclear burning. As a result, the fraction of the $\mathrm{C}$ and $\mathrm{O}$ layers that are ejected varies strongly for a given explosion energy and is rather sensitive to mass and explosion energy. Currently there is no good first principle calculations providing reliable predictions for explosion energies for a given stellar structure for masses above $15 M_{\odot}$.

Our new 3D LTE values provide definite constraints on the $[\mathrm{O} / \mathrm{C}]$ ratio instead of only an upper limit. The new lower value of $[\mathrm{Fe} / \mathrm{H}]$ derived here strengthens the constraint not to eject iron group elements from the inner core of the progenitor star. This limits the explosion energy and the magnitude of mixing due to Raleigh-Taylor instabilities during the SN explosion.

Ishigaki et al. (2014) have proposed an alternative scenario to explain the unique abundances in SM0313-6708. They investigated supernova yields of metal-free (Population III) stars and find that the high $[\mathrm{C} / \mathrm{Ca}]$ and $[\mathrm{C} / \mathrm{Mg}]$ ratios and upper limits of other elemental abundances are well reproduced with the yields of core-collapse supernovae (which have normal kinetic energies of explosion of $1 \mathrm{~B}$ ) and hypernovae (10 B) of Population III $25 M_{\odot}$ or $40 M_{\odot}$ stars. Their best-fit models assume that the explosions undergo extensive matter mixing and fallback, leaving behind a black hole remnant. In these models, $\mathrm{Ca}$ is produced by static/explosive $\mathrm{O}$ burning and incomplete $\mathrm{Si}$ burning in the Population III supernova/ hypernova, in contrast to our suggestion that the $\mathrm{Ca}$ originates from the hot-CNO cycle during pre-supernova evolution, which is, remarkably, consistent with the present data.

Although Ishigaki et al. (2014) well fits the observed abundances, their modeling uses several independent free parameters. For example, their models require significantly more mixing than is consistent with hydrodynamical simulations of Population III stars (Joggerst et al. 2009) and the fallback is adjusted independently of the explosion energy. Jetted or asymmetric explosions may be a possibility; however, this is another free parameter. In the Heger \& Woosley (2010) models the mixing is consistent with hydrodynamical simulations of Joggerst et al. (2009) and the fallback is computed selfconsistently with the explosion energy. Fuller discussion of the abundances of SM0313-6708 and SN modeling will be presented in a future paper.

\section{CONCLUSIONS}

The ultraviolet spectrum of SM0313-6708 is dominated by the lines of the $\mathrm{OH} \mathrm{A}-\mathrm{X}$ band, which are noticeably stronger than the $\mathrm{CH} C-\mathrm{X}$ lines. The new line lists of Masseron fit the $\mathrm{OH}$ and $\mathrm{CH}$ lines very well in both wavelength and strength. We could detect no lines of ${ }^{13} \mathrm{CH}$, which places a limit of more than 40 on ${ }^{12} \mathrm{C} /{ }^{13} \mathrm{C}$, and no lines of $\mathrm{NH}$ putting nitrogen at least two orders of magnitude less than $\mathrm{C}$ and $\mathrm{O}$. This supports there having been no mixing from the H-burning shell to the surface within SM0313-6708 and there being little nitrogen relative to $\mathrm{C}$ and $\mathrm{O}$ in the material from which SM0313-6708 formed. The clear lack of any metal lines apart from $\mathrm{Mg}$ I in the UVES ultraviolet spectrum also enabled us to lower the abundance limits for those elements whose strong lines are normally seen in this region, including $\mathrm{Fe}$, for which we now derive an $[\mathrm{Fe} / \mathrm{H}]$ upper limit of -7.52 dex $(3 \sigma)$ from $\langle 3 D\rangle$ LTE analysis. We derived an $[\mathrm{O} / \mathrm{C}]$ ratio of +0.02 with a $1 \sigma$ uncertainty of 0.175 dex for SM0313-6708, updated abundances measurements for $\mathrm{Mg}$ and $\mathrm{Ca}$, and derived new upper limits for nitrogen, the Fe-group elements and some of the alphaelements. These abundances remain consistent with a single massive Population III progenitor in the mass range 

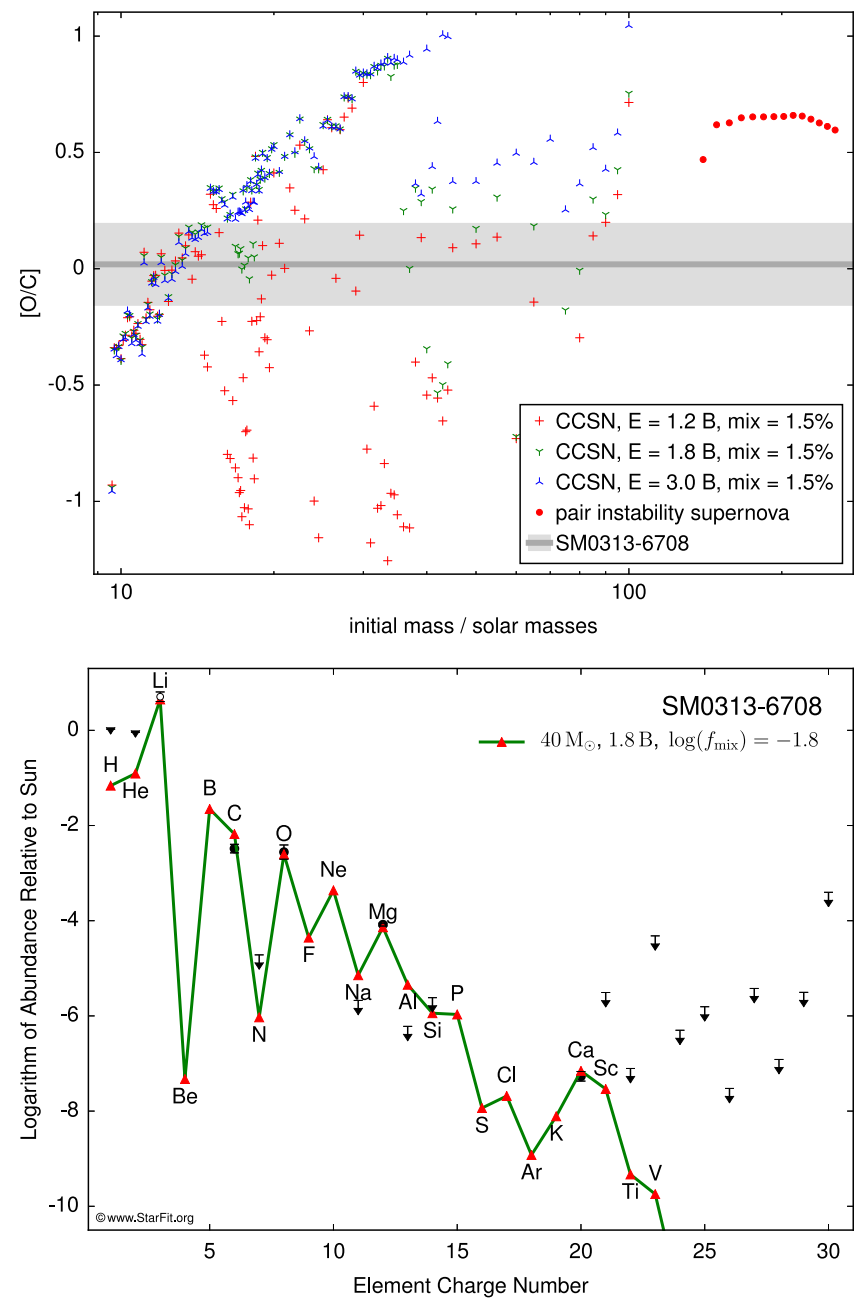

Figure 4. Top: range of $[\mathrm{O} / \mathrm{C}]$ abundances produced in a range of core collapse supernovae for three different explosion energies, $1.2 \mathrm{~B}, 1.8 \mathrm{~B}$, and $3 \mathrm{~B}$ and mixing of $1.5 \%$ (of helium core size; see Heger \& Woosley 2010). The dark gray line and the light gray shading show the derived SM0313-6708 and its $1 \sigma$ error bar of 0.175 dex. Red dots show the $[\mathrm{O} / \mathrm{C}]$ ratios for Pop III pairinstability supernovae (Heger \& Woosley 2002). Bottom: the yields from stars in the $40 M_{\odot}-60 M_{\odot}$ range provide the best fit to the observed abundance pattern of all the elements (see the main text). The $40 M_{\odot}$ fit is shown here.

$40 M_{\odot}-60 M_{\odot}$, low mixing, and modest explosion energies; the $\mathrm{Ca}$ production remains consistent with an origin from hydrostatic hydrogen burning. Improved upper limits on intermediate-mass elements, in particular $\mathrm{Na}$ and $\mathrm{Al}$, will further help constrain the models in the future. The strong Fe II lines in the vacuum UV between 2340 and $2625 \AA$ should allow one to constrain-or detect-the iron abundance of SM0313-6708 down to as low as $[\mathrm{Fe} / \mathrm{H}]=-9$.

A.H. was supported by an Australian Research Council (ARC) Future Fellowship (FT120100363). R.C. is supported by an ARC Discovery Early Career Researcher Award (DE120102940). A.C. and T.M. have been supported by the European Union FP7 programme through ERC grant number 320360. A.F. acknowledges support from NSF Career grant AST-1255160. This research been supported, in part, by ARC Discovery Project grants DP120101237 and DP150103294 and NSF grant PHY-1430152 (JINA-CEE). Facility: ESO(UVES)

\section{REFERENCES}

Aoki, W., Frebel, A., Christlieb, N., et al. 2006, ApJ, 639, 897

Alvarez, R., \& Plez, B. 1998, A\&A, 330, 1109

Asplund, M., Grevesse, N., Sauval, A. J., \& Scott, P. 2009, ARA\&A, 47, 481 Bernstein, R., Shectman, S. A., Gunxnels, S. M., Mochnacki, S., \& Athey, A. E. 2003, Proc. SPIE, 4841, 1694

Bessell, M. S., Christlieb, N., \& Gustafsson, B. 2004, ApJL, 612, L61

Cayrel, R., et al. 2004, A\&A, 416, 1117

Christlieb, N., Gustafsson, B., Korn, A. J., et al. 2004, ApJ, 603, 708

Collet, R., Asplund, M., \& Trampedach, R. 2006, ApJ, 644, 121

Dekker, H., D’Odorico, S., Kaufer, A., Delabre, B., \& Kotzlowski, H. 2000, Proc. SPIE, 4008, 534

Frebel, A., Aoki, W., Christlieb, N., et al. 2005, Natur, 434, 871

Frebel, A., Christlieb, N., Norris, J. E., Aoki, W., \& Asplund, M. 2006, ApJL, 638, L17

Gillis, J. R., Goldman, A., Stark, G., \& Rinsland, C. P. 2001, JQSRT, 68, 225

Gustafsson, B., Edvardsson, B., Eriksson, K., et al. 2008, A\&A, 486, 951

Hayek, W., Asplund, M., Collet, R., \& Nordlund, Å. 2011, A\&A, 529, A158

Heger, A., \& Woosley, S. E. 2002, ApJ, 567, 532

Heger, A., \& Woosley, S. E. 2010, ApJ, 724, 341

Ishigaki, M. N., Tominaga, N., Kobayashi, C., \& Nomoto, K. 2014, ApJL, 792, L32

Joggerst, C. C., Woosley, S. E., \& Heger, A. 2009, ApJ, 693, 1780

Keller, S. C., Bessell, M. S., Frebel, A., et al. 2014, Natur, 506, 463

Kurucz, R. 2011, http://kurucz.harvard.edu/linelists/linesmol/nh.asc

Lambert, J., Josselin, E., Ryde, N., \& Faure, A. 2013, in Betelgeuse Workshop, ed. P. Kervella, T. Le Bertre, \& G. Perrin (EAS Publication Series, Vol. 60; Les Ulis, France: EDP Sciences), 111

Lind, K., Primas, F., Charbonnel, C., Grundahl, F., \& Asplund, M. 2009, A\&A, 503, 545

Masseron, T., Plez, B., van Eck, S., et al. 2014, A\&A, 571, 47

Nakamura, T., Umeda, H., Iwamoto, K., et al. 2001, ApJ, 555, 880

Nordlund, A., \& Galsgaard, K. 1995, in A 3D MHD Code for Parallel Computers, Tech. Rep. (Copenhagen: Niels Bohr Institute), http://www.astro.ku.dk/ / g g/Papers/MHD_code.ps.gz.

Nordlund, Å., Stein, R. F., \& Asplund, M. 2009, LRSP, 6, 2

Plez, B. 2012, Turbospectrum: Code for Spectral Synthesis, Astrophysics Source Code Library, record ascl:1205.004

Sukhbold, T., \& Woosley, S. E. 2014, ApJ, 783, 10 